\title{
Cerebrospinal fluid-lymphatic fistula causing spontaneous intracranial hypotension in a child with kaposiform lymphangiomatosis
}

\author{
Karl A. Soderlund ${ }^{1}$ (D) Mark D. Mamlouk ${ }^{1,2} \cdot$ Vinil N. Shah $^{1} \cdot$ Jarod L. Roland ${ }^{3} \cdot$ William P. Dillon $^{1}$ \\ Received: 24 February 2021 / Revised: 4 May 2021 / Accepted: 10 June 2021 / Published online: 20 July 2021 \\ (C) This is a U.S. government work and not under copyright protection in the U.S.; foreign copyright protection may apply 2021
}

\begin{abstract}
Spontaneous intracranial hypotension is an uncommon etiology of secondary headaches in children. We report a unique case of a girl with kaposiform lymphangiomatosis who developed postural headaches and imaging features of spontaneous intracranial hypotension without a spinal extradural collection. The girl underwent dynamic computed tomography myelography which revealed a cerebrospinal fluid (CSF)-lymphatic fistula related to a lymphatic malformation associated with the right T10 nerve. She underwent surgical ligation of the CSF-lymphatic fistula, resulting in resolution of the headaches. Spinal CSF-lymphatic fistulas are rare and have previously been reported in two patients with Gorham-Stout disease. The current report suggests that patients with systemic lymphatic anomalies who develop postural headaches should undergo evaluation for spontaneous intracranial hypotension and a CSF-lymphatic fistula. If discovered, surgical ligation is a potential treatment.
\end{abstract}

Keywords Cerebrospinal fluid · Cerebrospinal fluid leak · Cerebrospinal fluid-lymphatic fistula $\cdot$ Child · Kaposiform lymphangiomatosis $\cdot$ Lymphatic anomaly $\cdot$ Spontaneous intracranial hypotension

\section{Introduction}

Spontaneous intracranial hypotension presents a diagnostic and therapeutic challenge to neurosurgeons, neurologists and neuroradiologists, requiring a systematic, multidisciplinary approach to diagnosis and treatment. Spontaneous intracranial hypotension is secondary to spontaneous spinal cerebrospinal fluid (CSF) leaks and consists of three recognized types: dural tears, ruptured meningeal diverticula and CSF-venous fistulas. The latter, which is the most recently described, typically results from direct communication between a meningeal

Karl A. Soderlund

ksoderlund.md@gmail.com

1 Department of Radiology and Biomedical Imaging, University of California San Francisco, 505 Parnassus Ave., Room L352, San Francisco, CA 94143, USA

2 Department of Radiology, The Permanente Medical Group, Kaiser Permanente Medical Center, Santa Clara, CA, USA

3 Department of Neurological Surgery, University of California San Francisco, San Francisco, CA, USA diverticulum and a vein [1]. Spinal CSF-lymphatic fistulas, on the other hand, are a rarer entity, and have been only reported in two patients with Gorham-Stout disease [2, 3]. The purpose of this case report is to highlight CSF-lymphatic fistula as an etiology of spontaneous intracranial hypotension in a child with a different condition predisposing to lymphatic abnormalities. Additionally, we emphasize the importance of considering spontaneous intracranial hypotension as a diagnosis in patients with systemic lymphatic anomalies presenting with specific headache syndrome and with characteristic imaging features.

\section{Case report}

A 9-year-old girl with a complex medical history presented with several years of chronic headaches. She was born full term. At age 7 months, she was diagnosed with kaposiform lymphangiomatosis with Kasabach-Merritt phenomenon and was treated with sirolimus after presenting with neck, mediastinal, hepatic and splenic masses and consumptive coagulopathy (Fig. 1). At age 9, after having chronic headaches initially attributed to migraine headaches for several years, the patient developed a horizontal diplopia due to an abducens 


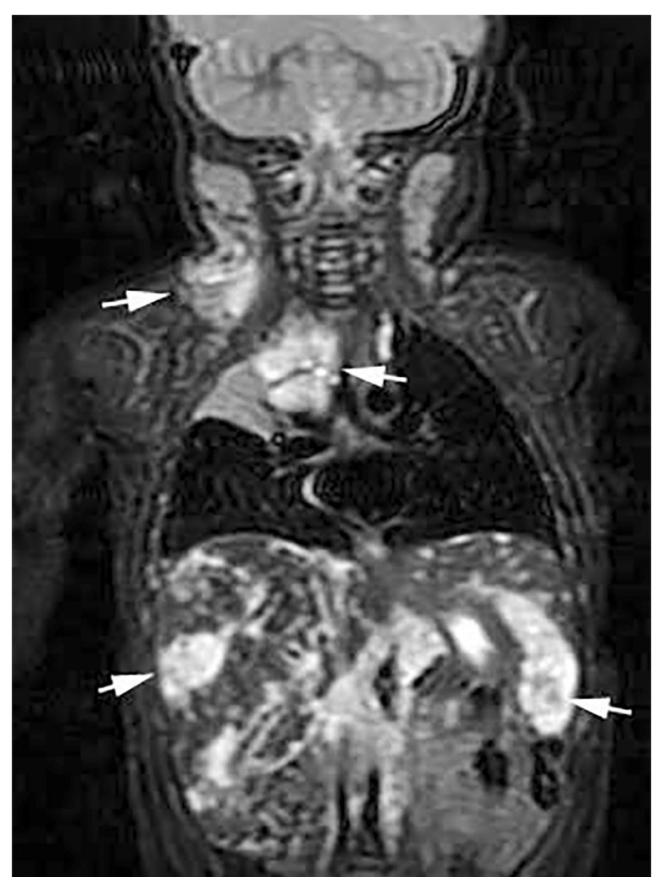

Fig. 1 Kaposiform lymphangiomatosis at 7 months old. Coronal fatsuppressed T2-weighted MR image shows multiple T2 hyperintense lesions (arrows) in the neck, mediastinum, liver and spleen. These lesions did not enhance after contrast administration (not shown) and were compatible with lymphatic malformations

nerve palsy and further history revealed that her headaches improved with lying supine. Non-contrast brain magnetic resonance imaging (MRI) was obtained; the patient could not receive gadolinium contrast material because of previous renal injury. Her initial brain MRI revealed brainstem sagging, flattening of the pons against the clivus and effacement of the prepontine cistern, dural venous sinus engorgement and lowlying cerebellar tonsils (Fig. 2). Total spine MRI revealed no evidence of a spinal extradural fluid collection but did reveal an upper cervical cord syrinx thought to be secondary to CSF flow obstruction from chronically low-lying cerebellar tonsils (Fig. 3). Based upon the patient's persistent postural headaches, cranial nerve deficits, brain imaging findings and the lack of an extradural fluid collection on spine MRI, a CSFvenous fistula resulting in spontaneous intracranial hypotension was suspected. In order to accurately localize a CSFvenous fistula, the patient was scheduled for dynamic computed tomography (CT) myelography.

Dynamic CT myelography was performed with the patient placed in the prone position on the $\mathrm{CT}$ table with a HoverMatt (HoverTech International, Allentown, PA) positioned under her hips. CT guidance was utilized to obtain intrathecal access at the L2-L3 level via a dorsal interlaminar approach with a spinal needle. Immediately following intrathecal administration of $6 \mathrm{cc}$ of preservative-free iohexol contrast (Omnipaque 240; GE Healthcare, Marlborough, MA), the HoverMatt was inflated, transiently raising the patient's hips for $10 \mathrm{~s}$ to

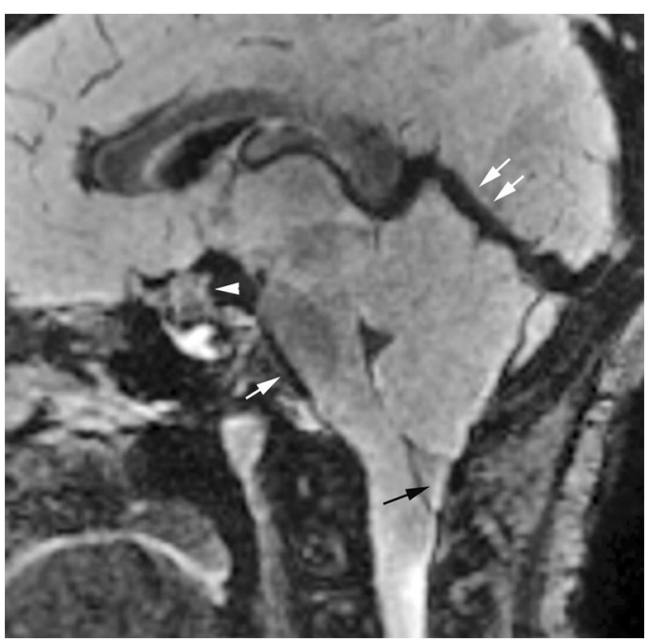

Fig. 2 Spontaneous intracranial hypotension at 9 years old. Midline sagittal fluid-attenuated inversion recovery (FLAIR) MR image of the brain shows typical features of spontaneous intracranial hypotension, including brain sagging with flattening of the pons (single white arrow), tonsillar ectopia (black arrow), dural venous sinus engorgement (double white arrows) and pituitary engorgement (arrowhead)

facilitate passage of intrathecal contrast into the thoracic and cervical spine. The HoverMatt was deflated and a CT scan of the entire spine immediately thereafter used a dynamic, multiphasic technique in which $\mathrm{CT}$ images were obtained from caudal to cranial then from cranial to caudal in two passes (CT

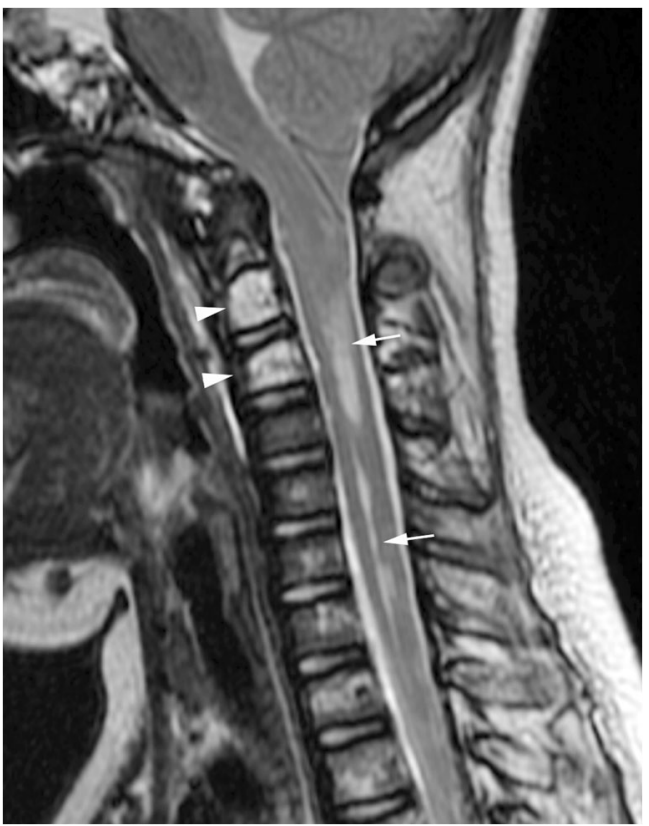

Fig. 3 Syrinx secondary to spontaneous intracranial hypotension. Midline sagittal T2-weighted MR image of the spine shows a cervical cord syrinx (arrows) secondary to long-standing cerebellar tonsillar herniation. No extradural spinal collection was identified. Multiple T2 hyperintense lesions in the cervical and upper thoracic spine are consistent with osseous involvement of kaposiform lymphangiomatosis (arrowheads at $\mathrm{C} 2$ and $\mathrm{C} 3$ ) 
dose index $=1.5 \mathrm{mGy}$ ). The dynamic CT myelogram revealed rapid contrast opacification of a CSF-lymphatic fistula associated with the right T10 meningeal diverticulum, which communicated with a lymphatic malformation in the right $\mathrm{T} 10$ T11 neural foramen and paraspinal space. The myelogram showed that the CSF-lymphatic fistula also communicated
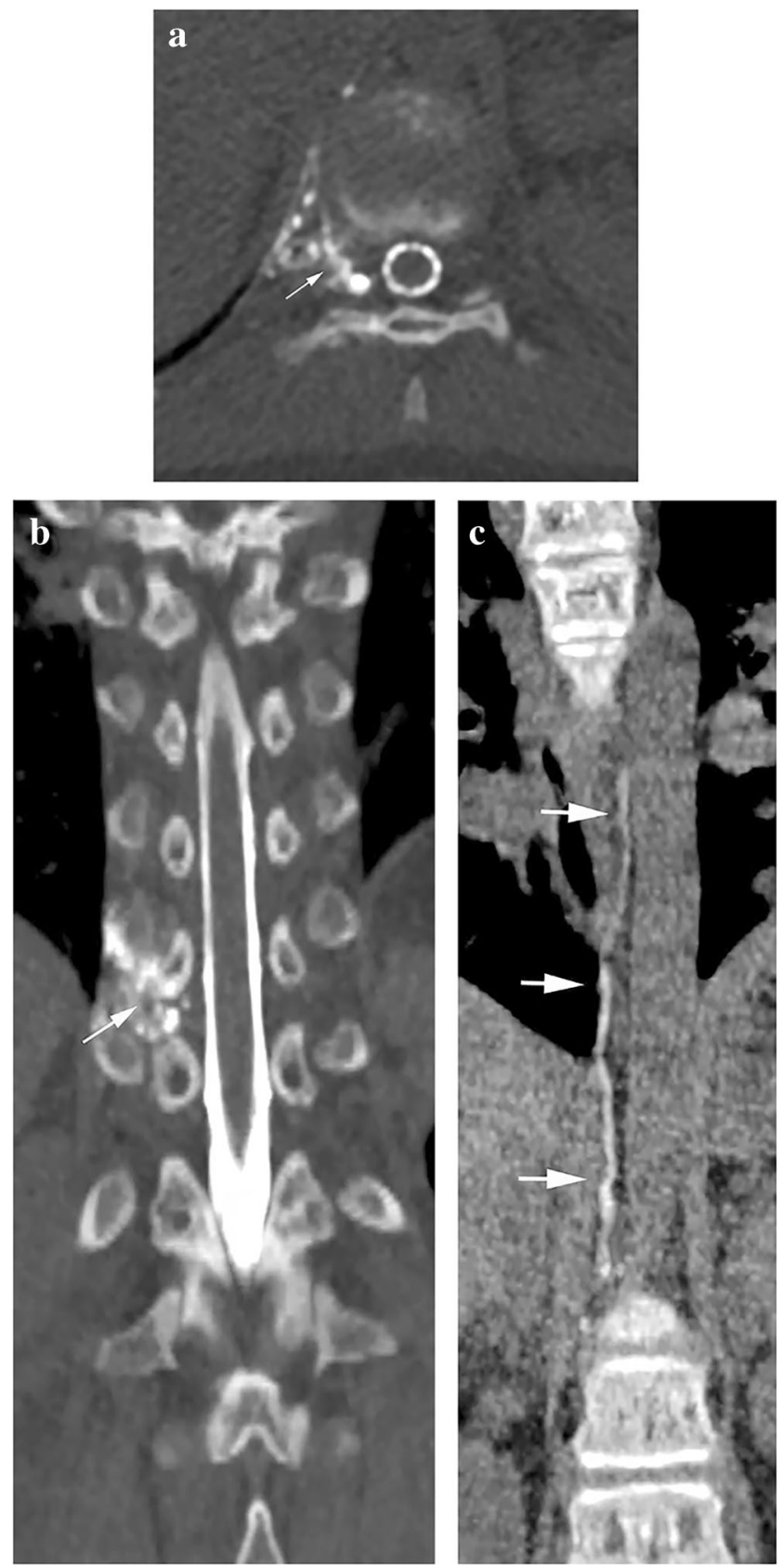

Fig. 4 Cerebrospinal fluid-lymphatic fistula detected on dynamic computed tomography (CT) myelogram. a, b Axial (a) and coronal (b) dynamic CT myelographic images show rapid egress of intrathecal contrast material from a meningeal diverticulum into a lymphatic structure in the right T10-T11 neural foramen and paraspinal soft tissues (arrows). c A coronal reformatted dynamic CT myelographic image demonstrates an opacified thoracic duct (arrows) to the level of its drainage into the left innominate vein, resembling a lymphangiogram with the thoracic duct, showing contrast opacification of the thoracic duct to the level of its drainage into the left innominate vein, resembling a lymphangiogram (Fig. 4). In retrospect, previous MR imaging showed that the right T10-T11 paraspinal lymphatic malformation (Fig. 5) likely communicated with the thoracic duct.

Targeted transforaminal epidural blood patching with $4 \mathrm{cc}$ of autologous blood, performed at the site of the CSFlymphatic fistula in the right T10-T11 neural foramen at the conclusion of the dynamic CT myelogram, transiently improved her headaches. Ultimately, because of persistent headaches, the patient underwent right T10-T11 foraminotomy and right T10 nerve root ligation to close the CSF-lymphatic fistula (Fig. 6). MR imaging of the brain performed 1 week after surgery revealed improved features of intracranial hypotension (Fig. 7). The patient experienced a transient rebound high-pressure headache attributed to changes in CSF dynamics related to CSF-lymphatic fistula ligation, which was treated with acetazolamide. The patient has been headache free for 15 months following surgery.

\section{Discussion}

Spontaneous intracranial hypotension is a treatable, often under-recognized etiology of headaches secondary to spinal leaks that requires a systematic, multidisciplinary approach to diagnosis and treatment. Spontaneous intracranial hypotension is typically a disease of adulthood and is rare in children. A series of 24 children with spontaneous intracranial hypotension at a tertiary referral center reported by Schievink and colleagues [4] in 2013 demonstrated that a generalized connective tissue disorder was present in slightly more than half.

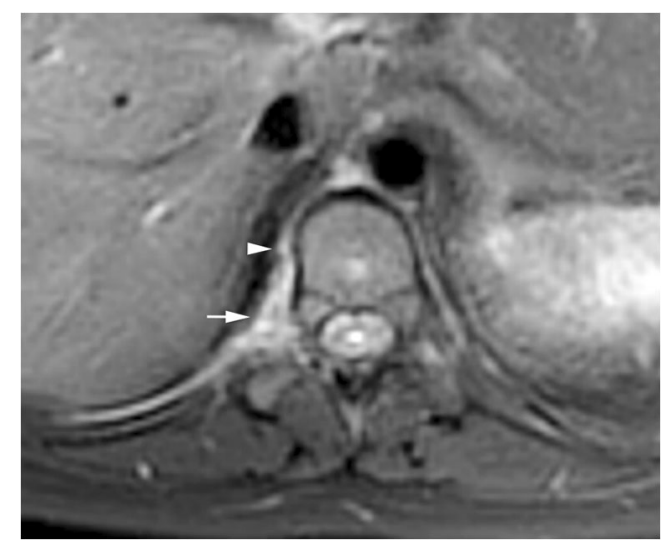

Fig. 5 Paraspinal lymphatic malformation. An axial fat-suppressed T2weighted image of the spine shows a right T10-T11 paraspinal hyperintense lesion (arrow) consistent with a lymphatic malformation in the context of other known system lymphatic malformations. There is an additional linear structure anteriorly (arrowhead) that likely represents communication with the thoracic duct, as this opacified on the computed tomography myelogram 
Fig. 6 Surgical ligation of cerebrospinal fluid (CSF)lymphatic fistula. a An intraoperative photo shows the CSF-lymphatic fistula involving the nerve root (arrow) elevated with a right-angle forceps exposed through a T10-T11 foraminotomy. b This nerve root and fistula were completely divided (double arrow) to resolve the CSF leak
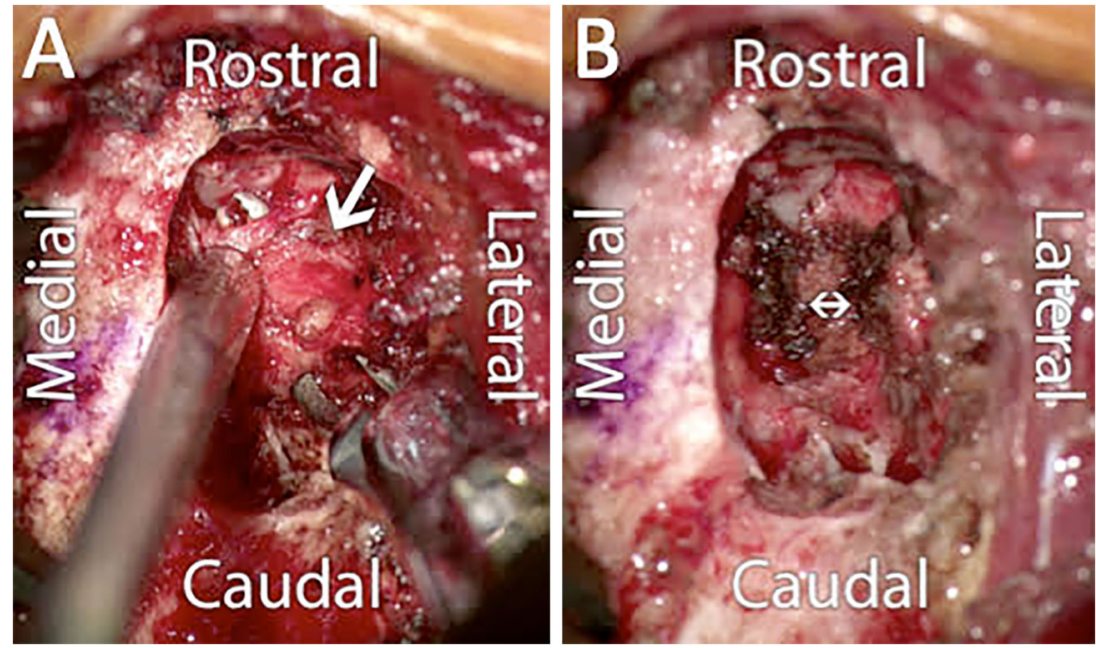

The rarity of spontaneous intracranial hypotension in the pediatric population makes the diagnosis even more difficult.

Because of increasing research on spontaneous intracranial hypotension, there is greater clarity on the different etiologies of spinal leaks. In 2016, Schievink et al. [5] devised a classification scheme for spontaneous spinal CSF leaks: dural tears (type 1), meningeal diverticula (type 2), CSF-venous fistula (type 3) and indeterminate/unknown (type 4). CSF-venous fistulas typically result from a direct connection between a meningeal diverticulum and a vein, resulting in continuous shunting of spinal fluid. CSF-venous fistulas were originally thought to be rare but probably constitute a quarter of spontaneous intracranial hypotension patients [1].

CSF-lymphatic fistulas, however, are exceedingly rare. To our knowledge, only two case reports of CSF-lymphatic

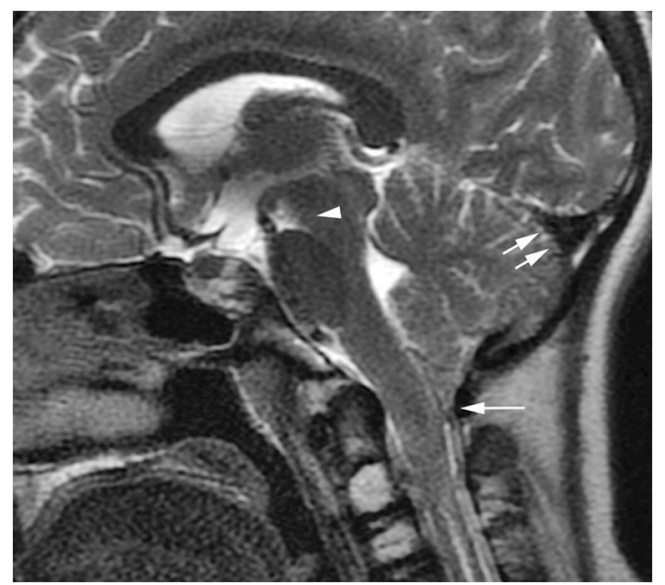

Fig. 7 Improved spontaneous intracranial hypotension after surgery. A midline sagittal T2-weighted image of the brain performed 1 week after ligation of the cerebrospinal fluid-lymphatic fistula demonstrates improved imaging features of spontaneous intracranial hypotension characterized by slightly diminished tonsillar ectopia (single arrow), normal triangular morphology of the transverse sinus (double arrows) and near normalization of the pontomesencephalic angle (arrowhead) fistulas have been previously reported in the literature, and both occurred in patients with Gorham-Stout disease [2, 3]. The first case involved a 1-year-old girl who had headaches and underwent surgical decompression for a presumed Chiari I malformation. She had persistent headaches and was ultimately found to have a CSF-lymphatic-osseous fistula, which resulted in spontaneous intracranial hypotension and tonsillar ectopia. This patient's CSF-lymphatic fistula was treated with percutaneous $n$-butyl cyanoacrylate glue embolization and resolved her symptoms for many years. We postulate that the underlying etiology of her CSF-lymphatic fistula is the result of dural erosion by adjacent intraosseous angioproliferative disease [2]. The second case involved a 30-year-old man who presented with intractable headaches and was ultimately diagnosed with a spinal CSF leak related to a fistula between a thoracic nerve root and an osteolytic lesion in his thoracic spine [3]. Other publications have also reported an "association" of Chiari I as well as skull base CSF leaks related to osteolytic lesions in patients with Gorham-Stout disease [2, $3,6]$. It is possible that many of the previously reported cases of acquired Chiari I malformations in the setting of GorhamStout disease may actually represent brain sagging and tonsillar ectopia secondary to spontaneous intracranial hypotension and a CSF-lymphatic fistula, as in our case. Furthermore, syrinx does not always suggest a Chiari I malformation. Spontaneous intracranial hypotension is a known cause of syrinx development related to brain sagging and tonsillar ectopia, as in our case, and should be recognized to avoid unnecessary foramen magnum decompression [7].

Like the patients reported by Adler et al. [2] and Suero Molina et al. [3], our patient also had a preexisting systemic lymphatic anomaly in the form of kaposiform lymphangiomatosis. Kaposiform lymphangiomatosis is an aggressive form of a generalized lymphatic anomaly of children characterized histopathologically by spindled lymphatic endothelial cells accompanied by malformed lymphatic channels and 
can occur in the thorax, bone marrow, abdominal viscera, peritoneum and soft tissues [8]. While the pathophysiology of development of the CSF-lymphatic fistula in our patient remains unknown, we postulate that the etiology is related to invasion of dura from the underlying lymphatic anomaly affecting transdural lymphatic channels. As such, patients with systemic lymphatic anomalies or paraspinal lymphatic malformations who present with orthostatic headaches should be considered for work-up for spontaneous intracranial hypotension. If brain imaging features suggest spontaneous intracranial hypotension, a high index of suspicion for CSF-lymphatic fistula should be maintained, and further evaluation should proceed in a similar manner to that of a CSF-venous fistula.

Imaging is central to the diagnosis and subsequent management of spontaneous intracranial hypotension. Initial imaging of patients with spontaneous intracranial hypotension should consist of a contrast-enhanced brain MRI as well as a noncontrast MRI of the entire spine with fat-suppressed T2weighted images. Brain imaging findings of spontaneous intracranial hypotension include brain sagging, tonsillar ectopia, flattening of the pons against the clivus, pituitary and dural venous sinus engorgement, smooth diffuse pachymeningeal enhancement and subdural collections. Administration of gadolinium contrast material aids in identifying diffuse pachymeningeal enhancement and dural venous sinus engorgement. The purpose of the initial total spine MRI is to evaluate for extradural fluid collections, the presence of which will guide subsequent dynamic CT or digital subtraction myelography to localize the site of leak. While there is typically an attempt to avoid ionizing examinations in the pediatric population whenever possible, $\mathrm{CT}$ myelography was necessary to identify the CSF-lymphatic fistula and can be performed with a low CT dose index, as in our case. Epidural blood patching does not generally result in lasting symptomatic relief for CSF-venous and CSF-lymphatic fistulas. Thus, if a CSF-lymphatic fistula is discovered, patients should be referred for neurosurgical management of the fistula.

\section{Conclusion}

Spontaneous intracranial hypotension is an uncommon etiology of headaches in children and is often related to an underlying generalized connective tissue disorder resulting in an intrinsic dural abnormality. A spinal CSF-lymphatic fistula, a much less common fistula compared to the better-known
CSF-venous fistulas, was the cause of spontaneous intracranial hypotension in our patient with a systemic lymphatic anomaly and has only been reported in the setting of Gorham-Stout disease. These findings suggest that patients with systemic lymphatic anomalies or paraspinal lymphatic malformations who present with orthostatic headaches should undergo workup for spontaneous intracranial hypotension and a CSFlymphatic fistula. If detected, CSF-lymphatic fistulas could be treated with surgical ligation.

Acknowledgments The content of this manuscript was presented as an electronic exhibit/excerpta at the American Society of Neuroradiology 58th Annual Meeting, May 30 to June 4, 2020 (virtual meeting due to the coronavirus disease 2019 [COVID-19] pandemic).

\section{Declarations}

Conflicts of interest None

\section{References}

1. Schievink W, Maya MC, Moser FG et al (2019) Spontaneous spinal CSF-venous fistulas associated with venous/venolymphatic vascular malformations: report of 3 cases. J Neurosurg Spine 32:305-310

2. Adler F, Gupta N, Hess CP et al (2011) Intraosseous CSF fistula in a patient with Gorham disease resulting in intracranial hypotension. AJNR Am J Neuroradiol 32:E198-E200

3. Suero Molina EJ, Niederstadt T, Ruland V et al (2014) Cerebrospinal fluid leakage in Gorham-stout disease due to dura mater involvement after progression of an osteolytic lesion in the thoracic spine. $\mathrm{J}$ Neurosurg Spine 21:956-960

4. Schievink WI, Maya MM, Louy C et al (2013) Spontaneous intracranial hypotension in childhood and adolescence. J Pediatr 163: 504-510

5. Schievink WI, Maya MM, Jean-Pierre S et al (2016) A classification system of spontaneous spinal CSF leaks. Neurology 87:673-679

6. Yoshimoto S, Takai K, Takahashi K et al (2018) Intracranial hypotension and hypertension: reversible Chiari malformation due to dynamic cerebrospinal fluid abnormalities in Gorham-Stout disease. Case report. J Neurosurg Pediatr 22:508-512

7. Middlebrooks EH, Okromelidze L, Vilanilam GK et al (2020) Syrinx secondary to Chiari-like tonsillar herniation in spontaneous intracranial hypotension. World Neurosurg 143:e268-e274

8. Croteau SE, Kozakewich HPW, Perez-Atayde AR et al (2014) Kaposiform lymphangiomatosis: a distinct aggressive lymphatic anomaly. J Pediatr 164:383-388

Publisher's note Springer Nature remains neutral with regard to jurisdictional claims in published maps and institutional affiliations. 